\title{
Simple method to evaluate the pixel crosstalk caused by fringing field effect in liquid-crystal spatial light modulators
}

\author{
Ignacio Moreno ${ }^{1,2^{*}}$ (D), María Del Mar Sánchez-López ${ }^{1,3}$, Jeffrey A. Davis ${ }^{4}$ and Don M. Cottrell ${ }^{4}$
}

\begin{abstract}
In this work we provide a simple experimental method to measure and evaluate the pixel crosstalk in phase-only liquid-crystal displays caused by the fringing field effect. The technique is a reverse engineering method that does not require information about the microscopic physical parameters of the liquid-crystal material or details of the fabrication and electronics of the display. Instead, it is based on the overall effect on the diffraction efficiency of displayed binary phase gratings as a function of the addressed gray level. We show how the efficiency of the zero (DC) and first diffraction orders provides valuable information enough to identify and quantify the pixel crosstalk. The technique is demonstrated with a modern phase-only liquid-crystal on silicon (LCOS) spatial light modulator (SLM), illustrating the limitations that this effect imposes to the spatial resolution of the device and providing quantitative measurement of the impact on the diffraction efficiency.
\end{abstract}

Keywords: Liquid-crystals displays, Diffraction gratings, Phase modulation, Fringing

\section{Introduction}

Spatial light modulators (SLM) are opto-electronic micro-displays capable to modulate the amplitude, the phase, or the state of polarization of light waves in space and in time [1]. The two well-stablished technologies nowadays are liquid-crystal devices (LCDs) and digital micro-mirror devices (DMDs). LCDs offer the great advantage of directly producing phase and polarization modulation; however, their response depends on the wavelengths and they are relatively slow devices, in the order of a few tens of $\mathrm{Hz}$ for nematic liquid crystals [2] or few $\mathrm{kHz}$ for ferroelectric liquid crystals [3]. On the contrary, DMDs are capable to operate at much larger framerates, reaching more than $30 \mathrm{kHz}$ and are wavelength insensitive; but they are binary amplitude SLMs and therefore phase modulation requires encoding

\footnotetext{
*Correspondence: i.moreno@umh.es

${ }^{1}$ Instituto de Bioingeniería, Universidad Miguel Hernández de Elche, 03202

Elche, Spain

2Departamento de Ciencia de Materiales, Óptica y Tecnología Electrónica,

Universidad Miguel Hernández de Elche, 03202 Elche, Spain

Full list of author information is available at the end of the article
}

techniques [4]. Newer devices with higher resolution, higher speed, or extended operational spectral ranges have led to progress in many different areas of optics \& photonics.

In this work we focus on phase-only LCDs. Ideally, they are pixelated linear retarders where the extraordinary phase can be varied controlled with the applied voltage. When the input beam is linearly polarized parallel to the liquid crystal director, they produce phase-only modulation useful for implementing phase-only diffractive elements and digital holograms $[5,6]$.

Modern technology mostly uses the liquid-crystal on silicon (LCOS) configuration [2]. The resolution and number of pixels is increasing rapidly for commercially available LCOS-SLMs. However, these LCOS displays suffer from different secondary effects that degrade their ideal optical modulation, which cannot be ignored when accurate response is required. These effects include flicker, which results in a phase fluctuation [7], external and multiple internal reflections [8], the aberration caused by the backplane deformation [9], or the pixel crosstalk caused 
by the fringing effect [10]. In all cases, the evaluation of these effects can be done at two different levels: (a) from the manufacturing point of view these effects should be minimized to reduce its impact on the devices, and (2) from the user's point of view, these effects should be measured and evaluated and, if possible, compensated. While most SLM commercial suppliers nowadays provide technical information about the phase fluctuation, the reflectivity and the uniformity of the devices, usually there is not information about the pixel crosstalk caused by the fringing effect, despite this is an effect that has been reported for many years [11]. The purpose of this paper it to provide a fast and simple method that could be used by users of commercial devices, who do not have access to fabrication details, to evaluate the impact on the fringing effect in phase-only SLMs.

Phase-only SLMs employ nematic liquid crystals in a parallel-aligned (PAL) configuration. In these cases, an electric field is applied to an area defined by the addressing electronics. The applied electric field tilts the liquid crystal molecules causing their optical phase to decrease. However, the electric field also affects the areas assigned to neighboring pixels, reducing the actual resolution. These effects are especially detrimental when the full SLM spatial resolution must be exploited, as we recently showed in the context of a complex amplitude encoding technique [12] that uses Nyquist diffraction gratings, i.e. gratings with 2 pixels per period.

As mentioned before, these fringing effects were reported years ago [11] in the original liquid crystal light valves (LCLVs) that were applied in pioneering optical data processing systems $[13,14]$. Then the industry developed electrically addressed LC-SLMs where the liquid crystal was confined by the two-dimensional grid of addressing electronics [15]. While this reduced the fill factor for each pixel because the transparent area of the pixel was reduced, these transmissive devices did not indicate severe problems caused by these fringing effects. Of note, Nyquist two-dimensional gratings were studied using these devices with results that agreed with theory [16].

However, the industry changed to reflective LCOS devices [17] where the addressing electronics are on the back-reflecting plane of the device. These devices allow a higher throughput and higher resolution. However, fringing effects have been reported in these newer devices, thus limiting their application for encoding phase functions with high resolution [18]. The cause is similar to that with the original LCLV devices because there is no physical barrier between adjacent pixels. For instance, recently we examined an approach for encoding complexamplitude functions using Nyquist diffraction gratings [12, 18]. The advantage of this technique is that the spatial variation of the amplitude information can be reduced compared with other approaches that require blazed gratings $[19,20]$. However, the fringing effect does not allow exploiting the high resolution required for Nyquist diffraction gratings, reducing their effectiveness.

Some works have provided models of the pixel crosstalk caused by this effect, and how it affects the diffraction efficiency [21-27]. They typically employ sophisticated methods to calculate the spatial distribution of the electric field provided by the device electrodes, and the corresponding microscopic spatial distribution of the liquid crystal material. This depends on the characteristics of the material (dielectric constants, refractive indices and elastic constants) [21-24]. Some other works study the diffraction efficiency and provides methods to partially compensate the reduction caused by this effect [10, 25, 26].

Typically, these effects are not noticed when diffraction gratings with large periods are examined. In this situation, they do not harm the diffractive efficiency of blazed gratings where the fringing effect effectively smooths the stepped phase pattern displayed by the device [23-25]. Thus, the diffraction efficiency of digital holograms is not dramatically affected, especially if they do not show very high spatial frequencies $[10,25]$.

On the contrary, binary phase diffraction gratings are very sensitive to the fringing effect because it rounds out the sharp edges of the binary phase pattern [26, 27], and this implies notable differences in the Fourier transform pattern. This effect can be described as a spatial low filtering of the ideally addressed phase grating profile, which can be modelled as its convolution with a given broadening kernel accounting for the fringing field effect $[22,27]$. The computation or the experimental characterization of this kernel can be used to compensate the related crosstalk [27].

Here we discuss a very simple method to identify when the SLM is affected by fringing and to evaluate its impact on the device performance. Often the user of the SLM does not have information about the LC material physical parameters or does not have access to advanced simulation software packages useful to calculate the LC spatial distribution. The advantage of the proposed technique is that it is based only on simple diffraction efficiency experiments performed with binary gratings displayed on the device with different gray levels.

The phase modulation measured with a retardance measurement (placing the SLM between crossed/parallel polarizers) [28] is compared with the expected diffraction efficiency measured for binary phase-only gratings [29]. We observe two kinds of mismatch with respect to the ideal binary grating response: 1 ) The zero (DC) diffraction order does not recover the $100 \%$ efficiency when the phase difference is of $2 \pi$ and 2) the DC vanishes at a gray level that does not coincide the maximum efficiency of the first diffraction orders. These effects become much clearer when the grating has small periods, 
becoming very relevant at the Nyquist limit of two pixels per period.

It is shown that this behavior can be modelled with a phase grating with a smooth phase profile. We use a simple equation with two parameters (the maximum phase depth and a roundness factor) that explains the experimental diffraction patterns, ranging from that of a perfect binary phase grating to a limiting case of a sinusoidal phase grating [30], where the diffraction efficiency follows the Bessel function behavior. We show that this simple approach is compatible with a convolutional approach [27], and it can be used to derive its related kernel.

The paper is organized as follows: after this introduction, Section 2 describes two methods used to evaluate the phase modulation of the display, one based on the retardance measurement, and another based on the diffraction efficiency of displayed gratings. Then we show how the diffraction efficiency measurements show discrepancies when approaching gratings with small periods. In Section 3 we provide a simplified model that accounts for the roundness of the phase profile generated by the fringing effect and in Section 4 we compare the predictions of this model with the experimental data, in order to evaluate the impact of the fringing effect. The final section gives our conclusions to the work.

\section{Retardance versus phase grating measurements}

The experimental system is similar to that used previously [31]. Light from a laser diode with a wavelength of $\lambda=625 \mathrm{~nm}$ (Thorlabs CPS635R) is expanded and collimated and enters a non-polarizing cube beam-splitter and illuminates the LCOS-SLM with normal incidence. The light reflected from the SLM is then analyzed to calibrate the phase modulation properties of the display. In this work we use a Hamamatsu LCOS-SLM device (model X10468-01), with $792 \times 600$ pixels and a pixel spacing of $\Delta=20 \mu \mathrm{m}$.

The first measurement is a calibration of the retardance as a function of the addressed gray level. A linear polarizer is placed on the input beam to select linearly polarized light oriented at $45^{\circ}$ with respect to the LC director. The reflected beam is analyzed with a linear analyzer oriented parallel and crossed with respect to the input polarizer. We use two film polarizers (Thorlabs LPVISE200-A) useful in the visible range, with a 2" aperture mounted on rotatable mounts. The intensity is then measured with a detector (Newport 1818C). In this situation, the normalized relative crossed and parallel intensities are given by $i_{\perp}=\sin ^{2}(\Phi / 2)$ and $i_{\|}=\cos ^{2}(\Phi / 2)$ respectively, where $\Phi$ denotes the retardance provided by the LCOS-SLM [28].

Figure 1 shows the obtained experimental results. Here we address the entire screen of the SLM with a uniform gray level $g$, so these measurements are not affected by fringing. The gray level $g$ is varied in the usual range from 0 to 255 . The intensity measurements for crossed and for parallel polarizers are normalized to the addition of the two components. Fig. 1a presents these normalized results, which reproduce the expected sinusoidal behavior with a variation of more than one period, thus indicating a total retardance variation that exceeds $2 \pi$. From these experimental data we calculated the retardance versus gray level function $\Phi(g)$ that best fits them. The corresponding curves $i_{\perp}(g)$ and $i_{\|}(g)$ are plotted as continuous curves in Fig. 1a, achieving a quadratic relative error of less than $1.8 \%$ with respect to the experimental data. The corresponding retardance function $\Phi(g)$ is presented in Fig. 1b, showing a linear relation between retardance and gray level, with a relative retardance difference of $\Phi=\pi$ for a gray level $g=100$ and of $\Phi=2 \pi$ for a gray level $g=200$, for the operating wavelength of $\lambda=625 \mathrm{~nm}$.

A second technique extensively used to calibrate the phase modulation is based on the efficiency of binary phase diffraction gratings [29] or binary diffractive lenses [32] displayed in the SLM. In this technique a onedimensional binary grating is displayed with two gray levels, one that is selected fixed (typically with gray level $g=0$ ) and another one with variable gray level $g$. In the case of pure phase-only modulators, the relative intensities of the zero (DC) order and the first diffraction orders are given by.

$$
i_{0}=\cos ^{2}(\varphi / 2),
$$

and

$$
i_{1}=\left(4 / \pi^{2}\right) \sin ^{2}(\varphi / 2),
$$

where now $\varphi(g)=\Phi(g)-\Phi_{0}$ is the relative phase difference between the retardance $\Phi(g)$ for gray level $g$ and that $\varphi_{0}$ for the reference level $g=0$. We note that two dimensional gratings could also be used, but the one dimensional gratings are sufficient.

Figure 2 shows experimental captures of the diffraction patterns obtained for this kind of one-dimensional binary phase diffraction gratings for different gray levels. Here we add a lens with focal length of $40 \mathrm{~cm}$ on the reflected beam and focus the Fourier transform plane onto a CCD camera from Basler (model scA1390-17 fc, with $1390 \times 1038$ square pixels of $4.65 \mu m$ side). The input and output polarizers are now set parallel to the LC director to obtain a phase-only modulation response of the SLM. In these results in Fig. 2 we use gratings with a large period of $d=16$ pixels.

The images in Fig. 2 show the expected classical diffraction pattern of a binary phase grating, which evolve from a single DC order when $g=0$, reaches the maximum diffraction efficiency for $g=100$ where 


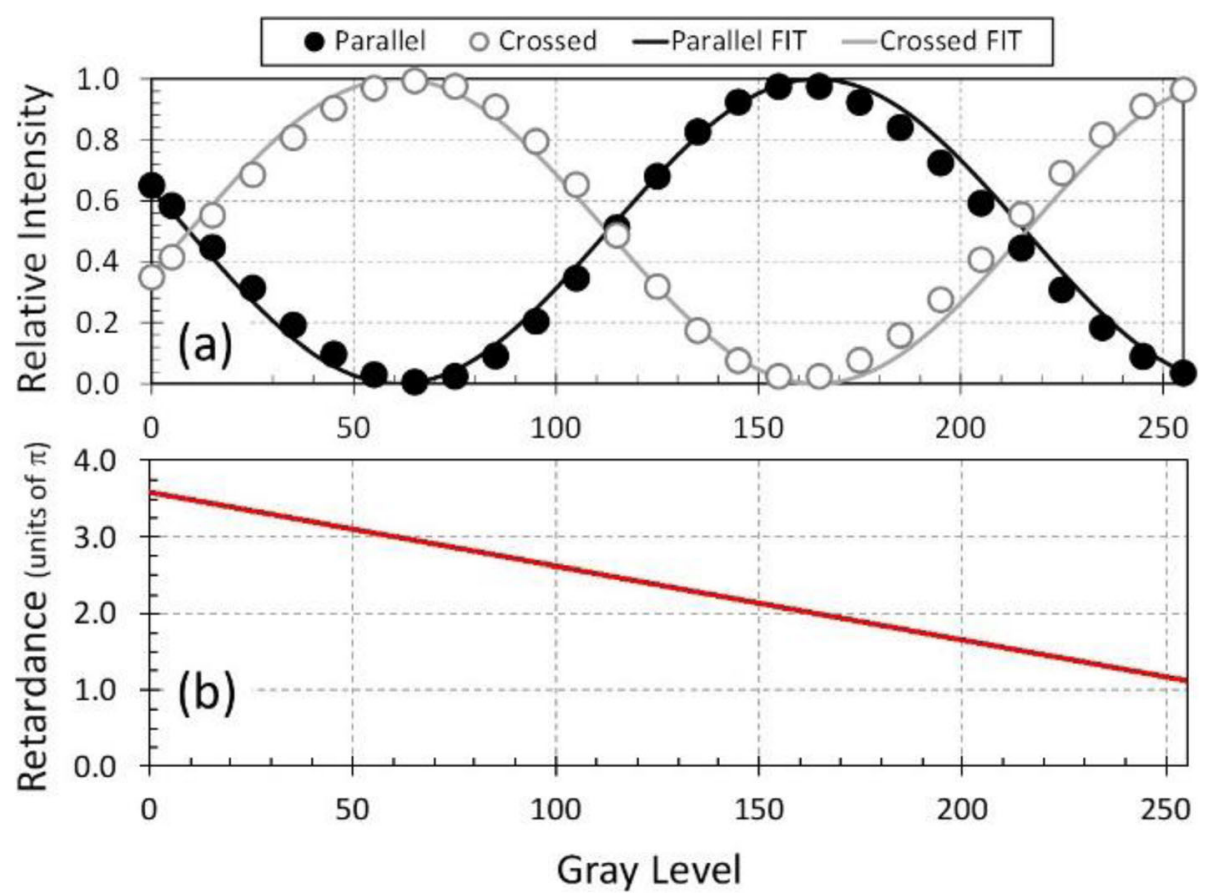

Fig. 1 (a) Experimental measured data (dots) for parallel and crossed polarizers as a function of gray level ( $g$ ) and best fit curves (continuous lines). (b) Retardance versus gray level $\Phi(g)$ that best fits the experimental data

the phase modulation is $\varphi=\pi$, and gets back to a single DC order for $g=200$, where the phase modulation is $\varphi=2 \pi$. These diffraction patterns in Fig. 2 show a null intensity for the even diffraction orders, as it is expected for the binary diffraction gratings with $1 / 2$ fill factor [33] (in this case the gratings have 8 pixels with $g=0$ and other 8 pixels with the variable gray level $g$ ). The results in Fig. 2 correspond to gratings displayed in vertical direction, but we did not notice any significant difference when the grating was displayed horizontally.

We note that we are using an LCOS-SLM with analog electronics, therefore free of flicker or phase fluctuation [34]. This is why the DC order can be practically cancelled when the phase difference $\varphi=\pi$ is reached for $g=$ 100. Nevertheless, some weak DC noise is still visible at this level due to some non-negligible reflection at the outer surface of the SLM.

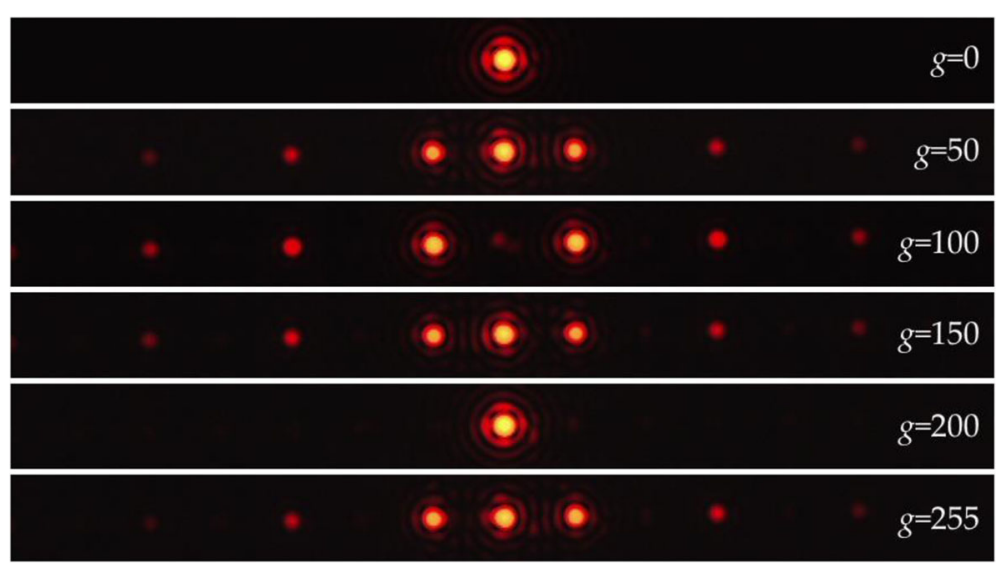

Fig. 2 Experimental diffraction patterns obtained for binary phase diffraction gratings with a period of $d=16$ pixels and different gray levels 
The situation changes drastically when the period of the grating is reduced. To illustrate the case, Fig. 3 shows the diffraction patterns captured when the binary phase grating is displayed with a period of only $d=2$ pixels, i.e. a Nyquist grating. This grating diffracts with much larger angles and therefore we require using a lens with much shorter focal length to capture the diffraction pattern with the same CCD sensor (the size of the Fourier transform scales proportional to the focal length). We used a photographic objective with focal length of $5 \mathrm{~cm}$. However, then, the focused diffraction orders appear in the captured images as very narrow sharp peaks. Therefore, in order to more clearly visualize the diffraction orders, we slightly defocused the Fourier transform plane.

The results in Fig. 3 show very different patterns compared to Fig. 2 and illustrate how the fringing is affecting the profile of the phase grating provided by the SLM. Three effects are clearly visible in the patterns in Fig. 3: a) the $\pm 2^{\text {nd }}$ diffraction orders are now clearly visible for gray levels greater than $g=100$; b) the DC order is no longer cancelled for the gray level $g=100$ and appears weaker for larger gray levels, and c) we never recover the situation with a single DC order that happens for the binary grating with $\varphi=2 \pi$ phase modulation.

We note here that the second diffraction order generated by this Nyquist grating coincides with the first diffraction order generated by the pixelated structure of the display. However, the Hamamatsu LCOS-SLM has a very high fill factor $(F)$ of more than $98 \%$ and the intensity of the central DC order generated by this pixelated structure is thus given by $F^{2}=96 \%$. Therefore, the contribution of the four first diffraction orders generated by the pixelated structure is less than $1 \%$ and this contribution can be ignored. As shown in Fig. 3, cases $g=0$ or $g=50$ do not show any significant second diffraction

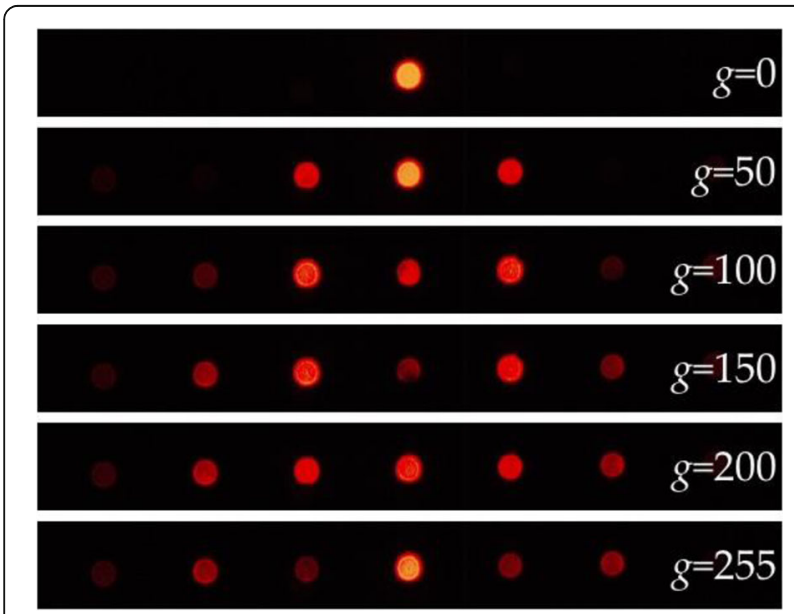

Fig. 3 Experimental diffraction patterns captured for binary phase diffraction gratings with a period of $d=2$ pixels and different gray levels order, and therefore the second orders observed for higher gray levels can be fully attributed to the fringing effect.

As it is shown next, all these effects can be explained by the roundness of the phase profile of the displayed binary diffraction grating caused by the fringing effect on the SLM. To quantify this effect, we conducted more precise experiments where we measure the intensities of the diffraction orders as a function of the addressed gray level. Nevertheless, the experimental comparison provided by Fig. 2 and Fig. 3 represent a very simple and fast procedure to identify when the SLM is affected by the fringing effect.

Figure 4 presents results of the intensities of the DC and first diffraction orders as a function of the phase difference $\varphi(g)$ in the binary phase grating. We change the period of the grating from a period of $d=10$ pixels down to the Nyquist case with a period of $d=2$ pixels. The experimental data are shown by the dots. In order to see the effects more evident, we also plot in all graphs the expected intensity $i_{0}=\cos ^{2}(\varphi / 2)$ and $i_{1}=\left(4 / \pi^{2}\right) \sin ^{2}(\varphi / 2)$ (Eqs. (1)), assuming the phase-shift to gray level calibration relation $\varphi(g)=\Phi(g)-\Phi_{0}$ given by Fig. 1(b).

When the grating has a period of $d=10$ pixels (Fig. 4a), the intensities of the diffraction orders agree reasonably well with the expected results. The first order reaches the maximum intensity around $\varphi=\pi$ and returns to zero around $\varphi=2 \pi$. However, even with this large period, there are some discrepancies which are most clearly evident in the DC order obtained for the high values of $\varphi(g)$. Here, the experimental curve does not match the theoretical relation; instead, it appears slightly shifted to higher values. In addition, the secondary maximum expected at $\varphi=2 \pi$ does not reach the maximum value.

These effects are more evident as the period of the grating decreases, as shown in Figs. $4 \mathrm{~b}, \mathrm{c}$ and $\mathrm{d}$. The values of the phase modulation (or equivalently the gray levels) that provide the minimum and the secondary maximum of the DC intensity increase as the period decreases; and the maximum intensity that the secondary maximum of the DC reaches is less intense as the period decreases.

The diffraction efficiency of the first diffraction order is less affected than the DC order. The experimental data follow well the expected curves when the grating has $d=10$ and $d=6$ pixels per period. However, discrepancies are again shown when $d=4$ and most clearly for the Nyquist grating, $d=2$. Here, again the experimental data look shifted to higher phase values. As mentioned before, these gratings should not produce the second diffraction order. However, for the Nyquist grating $(d=2)$ we also include the measurement of the intensity of this second diffraction order since it takes non-negligible values for high gray levels (Fig. 4d). 

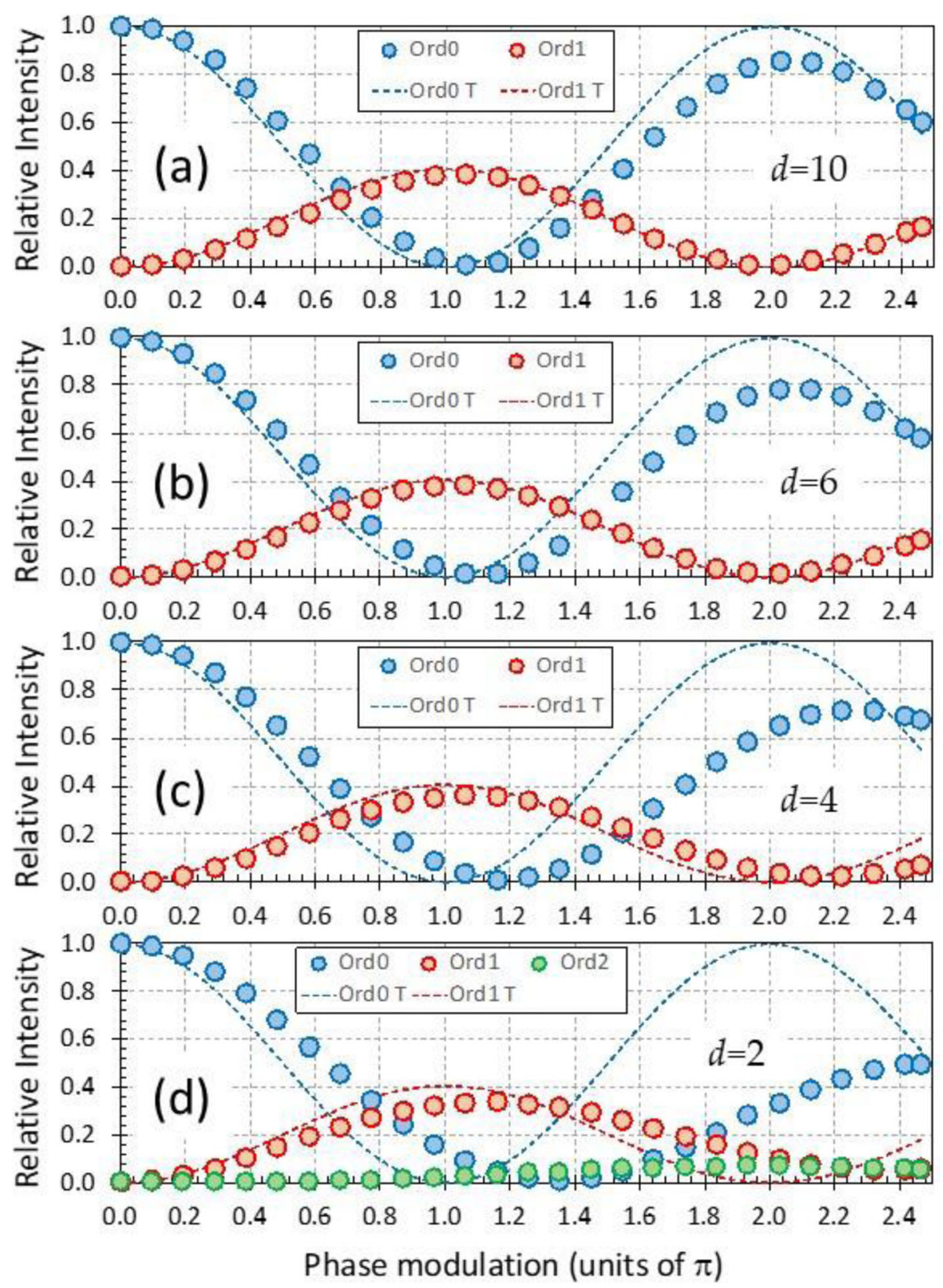

Fig. 4 Experimental intensities of the DC and first diffraction orders for displayed binary phase diffraction gratings with periods of $d=10,6,4$ and 2 pixels, as a function of the phase modulation $\varphi(g)$. The dotted lines correspond to the theoretical intensities

\section{Phase gratings with smooth rounded edges}

As stated earlier, the fringing effect basically smooths the binary grating phase profile, and this is the reason of the discrepancies in the resulting diffraction efficiency. To mathematically model the real phase profile $\varphi(x)$ of these gratings displayed by the SLM affected by fringing, we consider a function similar that used by Gori et al [35] to design continuous phase triplicator gratings with optimal efficiency, with the following relation:

$$
\varphi(x)=M \pi\left\{\frac{1}{C} \arctan [a \cos (\gamma x)]\right\}
$$

Here $\gamma=2 \pi / d$ and $d$ is the period of the grating. The numerical constant $a$ gives the roundness of the phase profile, and $C$ is a normalizing constant equal to the maximum value of the $\arctan ()$ function. The value of $C$ depends on the selected value of $a$ and it is included in Eq. (2) to make the function within the curly brackets 
range between -1 and +1 , thus making the phase grating profile $\varphi(x)$ range between $-M \pi$ and $+M \pi$. Therefore, the two parameters we use to describe the fringing effects are the roundness constant $a$ and the maximum phase difference $\varphi_{\max }=M 2 \pi$. This last parameter $M$ differentiates the profile in Eq. (2) from the triplicator design in [35].

Figure 5 shows some phase profiles derived with Eq. (1) for four different values of $a$. For large values the phase profile approaches the perfect binary phase square signal (Fig. 5a). As the value of $a$ decreases, the edges of the binary pattern get progressively smooth as shown in Fig. $5 \mathrm{~b}$ and $\mathrm{c}$ for $a=10$ and $a=2$ respectively. Values below $a=0.1$ makes the phase grating achieve a sinusoidal profile of the type $\varphi=\varphi_{\max } \cos (\gamma x)$. The two extreme situations, the binary phase profile and the sinusoidal phase profile, they both have analytical expressions for the intensities of the diffraction orders as a function of the maximum phase difference $\varphi_{\max }$. As mentioned above (Eqs. (1)), the binary grating has efficiencies $i_{0}=\cos ^{2}\left(\varphi_{\max } / 2\right)$ and $i_{1}=\left(4 / \pi^{2}\right) \sin ^{2}\left(\varphi_{\max } / 2\right)$ and there is no second order [25]. For the sinusoidal phase grating the intensities of the diffraction orders are given by $i_{m}=J_{m}^{2}\left(\varphi_{\max }\right)$ [30], where $J_{m}$ is the Bessel function of first kind and order $m$. In between, the phase profile changes in a non-linear way with the parameter $a$.

Figure 6 shows the intensities for the DC, first and second diffraction orders generated by the phase gratings shown in Fig. 5 as a function of the maximum phase variation $\varphi_{\max }$. We use the same four values $a=1000$, $a=10, a=2$ and $a=0.1$ as in Fig. 5. These intensities have been numerically calculated using standard Fourier transform methods, as described in [36]. The first case ( $a=1000$, Fig. 6a) reproduces the binary phase grating behavior. The effects displayed in the experimental data in Fig. 4 are clearly shown in Figs. 6b $(a=10)$ and $6 \mathrm{c}$ $(a=2)$. The maximum efficiency for the DC order moves to higher phase values and its intensity does not return to its maximum original value. Note for instance the resemblances of the results in Figs. $4 \mathrm{~d}$ and $6 \mathrm{~d}$.

The second diffraction order is negligible for $a=1000$ and for $a=10$ but starts to be relevant for $a=2$. Finally, the last case in Fig. $6 \mathrm{~d}(a=0.1)$ reproduces the Bessel functions expected from a sinusoidal phase grating [30].

In order to compare the experimental data in Fig. 4 with the above-described model, we look at the maximum and minimum values of the diffraction orders, as well as to the phase values where they are produced. To this aim, Fig. 7 shows another numerical calculation of how these values change with the parameter $a$. We present the values in the range from $a=500$ to $a=0.1$ in a logarithmic scale.

In Fig. $7 \mathrm{a}$ we plot the maximum intensity that reaches the DC order, the first order and the second orders for each value of $a$ (blue, red and green curve, respectively). In the case of the DC order, we plot the secondary maximum that happens for around the phase shift of $2 \pi$. For $a=500$, which approaches the binary grating, these values are as expected, practically of $100 \%, 40.5 \%$ and zero respectively. However, as the parameter $a$ decreases, we see that the fringing starts to impact these values.

The intensity of the secondary maximum of the DC order shows the greatest variation with $a$ (changing from $100 \%$ to about only $16 \%$ ), thus being the most sensitive experimental parameter to determine the effect in the experimental data. On the contrary, the maximum of the first diffraction order shows very little variation (from $40.5 \%$ to $33.9 \%$ ). The second order is null for high values of $a$, and reaches the maximum value of $23.6 \%$ when the sinusoidal phase grating is reached.

Fig. $7 \mathrm{~b}$ shows the theoretical phase modulation value $\varphi_{\max }$ that provides these maximum intensities at the corresponding diffraction order. The phase value that provides the minimum intensity of the zero order (black

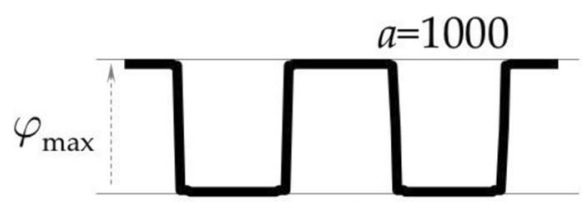

(a)

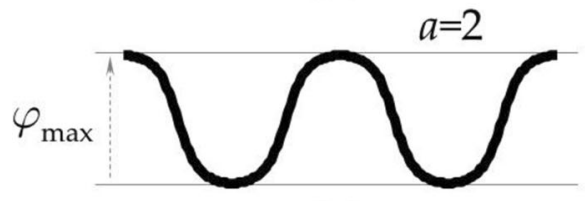

(c)

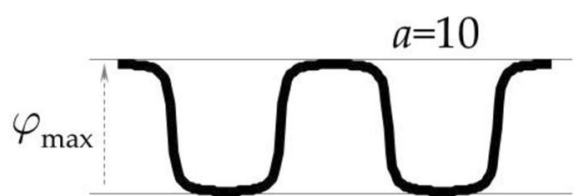

(b)

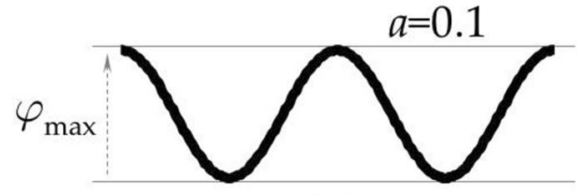

(d)

Fig. 5 Phase profiles for different values of the parameter $a$ in Eq. (1) 

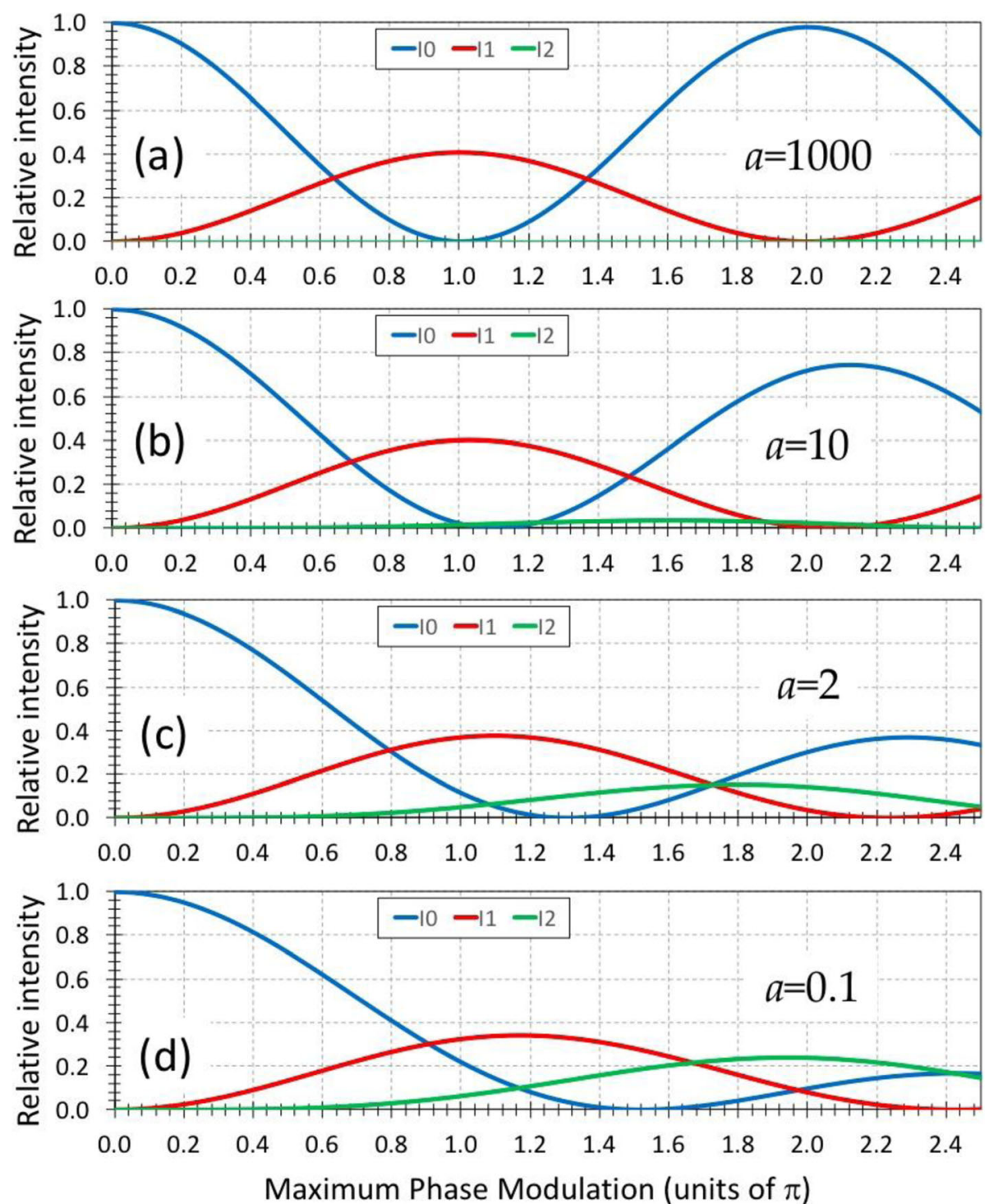

Fig. 6 Theoretical diffraction efficiency of the DC, first and second diffraction orders as a function of the maximum phase $\varphi_{\max }$ for $a=1000, a=$ $10, a=2$ and $a=0.1$

curve in Fig. 7(b)) is provided as well. Again, the expected values for the binary grating are obtained for $a=$ 500. The secondary maximum of the DC order is obtained for $2 \pi$, and the maximum value of the first order is obtained for $\pi$, and this coincides with the minimum of the DC order.

However, we observe the behavior shown in the experiments as the value of $a$ increases. The maximum in the DC order (blue curve in Fig. 7b) moves to higher phases, shifting almost by $\pi / 2$ when the sinusoidal phase grating is reached $(a=0.1)$. The minimum of the DC (black curve) is shifted with the same proportion but starting from the value of $\pi$. On the contrary, the maximum intensity of the first diffraction order (red curve) is shifted with much smaller value, as shown in the separation between the black and red curves in Fig. 7b. This explains the mismatch between the maximum efficiency at the first diffraction order and the minimum efficiency of the DC order that we observe in the experiments. Finally, the maximum intensity of the second order (green curve) suffers a shift like that observed in the DC order.

In the next section, we compare this model with the experimental data.

\section{Characterization of the LCOS-SLM device}

In order to characterize the fringing effect in the LCOSSLM device used in this work we use the experimental data shown in Fig. 4. As described in the previous section, the maximum efficiency value that the DC diffraction order reaches in the secondary maximum provides 

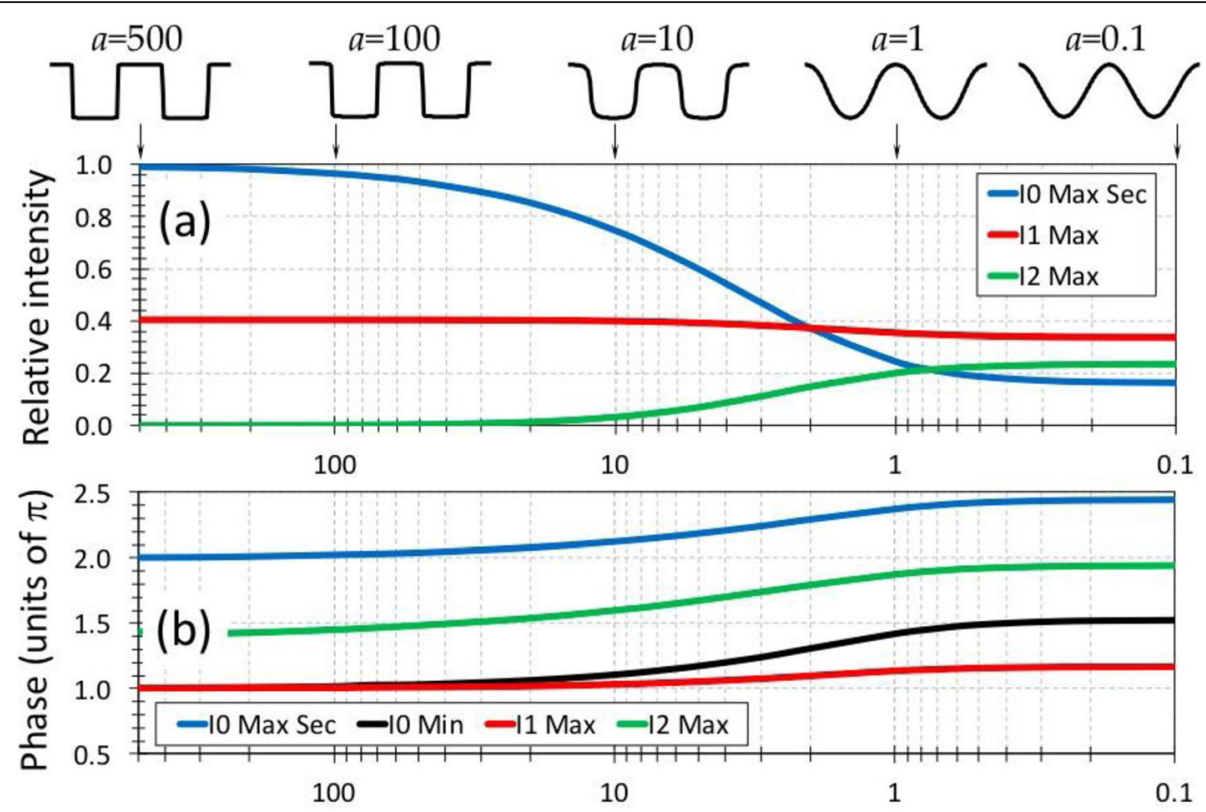

Parameter $a$

Fig. 7 a Maximum theoretical intensity of the DC, first and second diffraction orders as a function of the parameter $a$ (for the DC the value corresponds to the secondary maximum). $\mathbf{b}$ Phase value $\varphi_{\max }$ that provides the maximum efficiency of the DC order (blue curve), first order (red) and second order (green), and phase values that provides minimum intensity of the DC order (black), as a function of $a$

a measurement with good sensitivity to determine the value of the parameter $a$. Therefore, we determined the value of a for the four cases shown in Fig. 4 using the blue curve in Fig. 7a, leading to the values $a=20.2$ for the period of $d=10$ pixels, $a=12.2$ for $d=6$ pixels, $a=$ 8.8 for $d=4$ pixels and $a=3.4$ for the Nyquist grating with $d=2$ pixels per period. Then, we used this value in each case, together with the phase modulation to calculate the intensity of the corresponding diffraction order and compared the result with the experimental data. This comparison is provided in Fig. 8.

At an initial step, we select in all cases that the maximum phase $\varphi_{\max }(g)$ for each grating is the same value as given by the calibration $\varphi(g)=\Phi(g)-\Phi_{0}$ shown in Fig. 1b. This results in an excellent agreement in the whole gray level range for the gratings with $d=10$ and with $d=6$ pixels per period (see Figs. $8 \mathrm{a}$ and $\mathrm{b}$ ). However, for the shortest periods ( $d=4$ and $d=2$ ) we added a refinement to achieve the best results shown in Fig. 8, consisting in multiplying the value of $\varphi_{\max }(g)$ by a multiplicative factor that reduces the effective maximum phase modulation to $\varphi^{\prime} \max _{\text {max }}$. For $d=4$ this factor is of $94 \%$, while for of $d=2$ is of $89 \%$. This multiplicative factor for the phase modulation is required to achieve the excellent fit shown in Figs. 8c and d, especially for the higher gray levels. This multiplicative factor is a reasonable expected effect since the fringing-field effect has been also probed to reduce the effective phase modulation [26].
On top of each graph in Fig. 8 we draw the phase profile for each grating represented in eleven pixels of the SLM. These figures therefore illustrate the strength of the fringing effect in the phase profile. We note that the pixel pitch for the employed LCOS-SLM is of $\Delta=20 \mu \mathrm{m}$.

Finally, we show that this simplified approach is compatible with the convolutional approach in [22, 27]. For that purpose, we consider the ideal rectangular binary phase profiles $\vartheta(x)$, as shown in Fig. 9a. Then, for each profile we calculate its Fourier transform $\Gamma(p)=\mathcal{F}[\vartheta(x)]$, where $p$ denotes the spatial frequency. Then, the Fourier transform of each phase grating is multiplied by a function of the following form:

$$
H(p)=\exp \left[-\left(\frac{|p|}{\sigma}\right)^{m}\right] .
$$

This function represents the Fourier transform of the broadening kernel. If $m=2$ it corresponds to a Gaussian function. We consider the absolute value of $p$ in Eq. (3) since we experimentally observe a symmetric distribution of diffraction orders. However, asymmetric functions can be also considered in general, like in $[22,27]$ if an asymmetric distribution is observed.

To perform the calculations, each pixel of the SLM is oversampled with an arbitrary number of 50 subpixels. Then, $\Gamma(p)=\mathcal{F}[\vartheta(x)]$ is calculated for each binary phase grating $\vartheta(x)$ in Fig. 9a and the product $H(p) \Gamma(p)$ is 

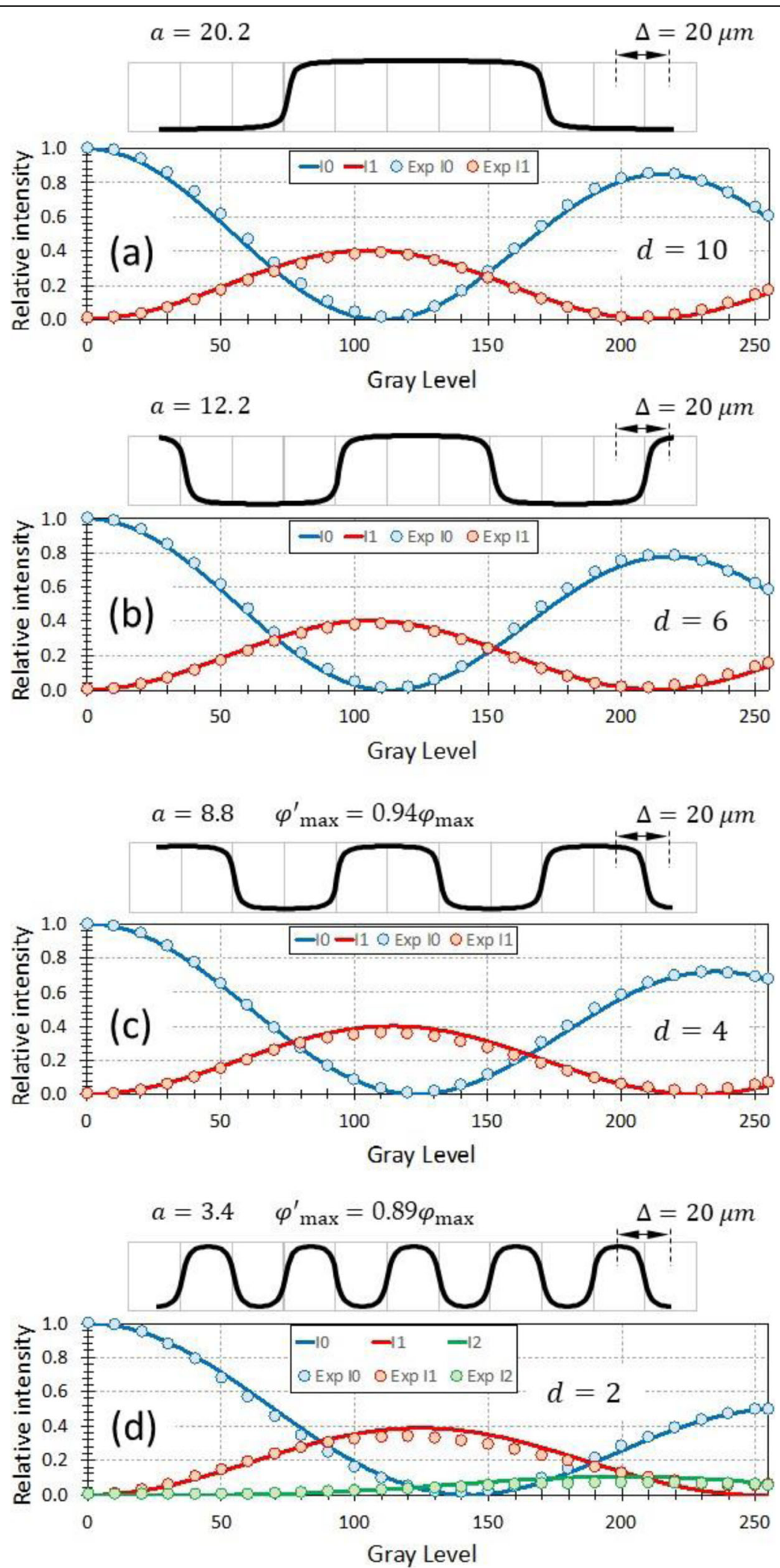

Fig. 8 Comparison of the experimental intensity at the diffraction orders (dots) with the theoretical values (continuous lines) expected for the corresponding grating with after calibrating the parameter a in each case. On top of each graph is drawn the corresponding phase profile $\varphi(x)$ of each grating 


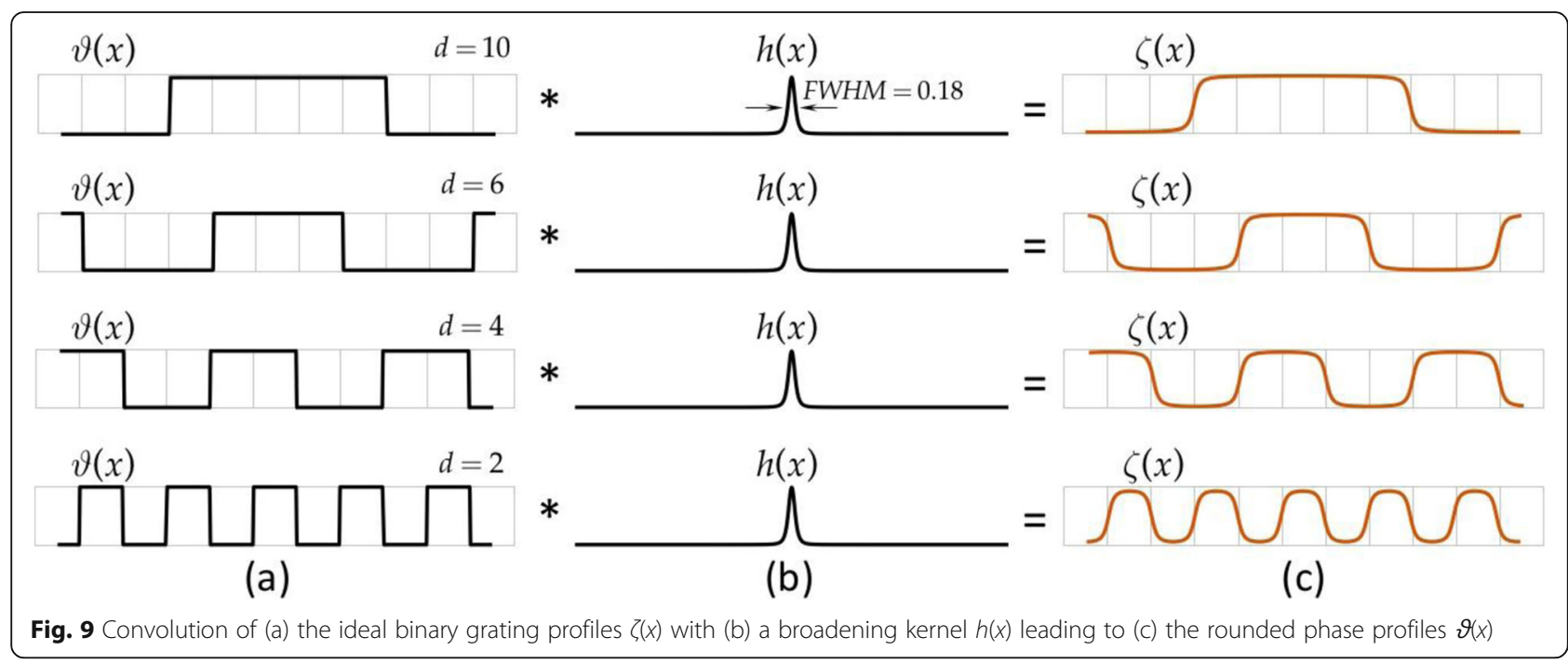

inverse Fourier transformed. Thus, a new function $\zeta(x)$ $=\mathcal{F}^{-1}[H(p) \Gamma(p)]=h(x) * \vartheta(x)$ is retrieved for each grating, where * denotes the convolution operation and where $h(x)=\mathcal{F}^{-1}[H(p)]$ is the broadening kernel. We seek for the values of $m$ and $\sigma$ in Eq. (3) that minimize the difference between the profiles $\zeta(x)$ and the corresponding phase profiles $\varphi(x)$ derived from the experimental data and shown in Fig. 8. The best results are obtained for values $m=1.21$ and $\sigma=0.74$ pixels. These values provide an excellent agreement for all four phase profiles, with an absolute error difference of less than $0.02 \%$ between $\zeta(x)$ and $\varphi(x)$ in all four gratings shown in Fig. 8. The corresponding broadening kernel $h(x)$ is shown in Fig. 9b, represented in the same scale as the phase profiles, and showing a full width at half maximum $F W H M=0.18$ pixels. The resulting phase profiles $\zeta(x)$ are shown in Fig. 9c, illustrating a behavior equivalent as that shown in Fig. 8.

\section{Conclusions}

This paper presents an approach that is important for users of LC-SLMs to quantify fringing-field effects based on diffraction efficiency experiments with displayed binary phase gratings with different periods. The goal of the method is not to determine the cause of the pixel crosstalk but to specify the overall response and its impact in terms of diffraction efficiency and spatial resolution, parameters that are relevant for users of the devices. Therefore, the technique does not require the computation of the spatial distribution of the director axis in the liquid-crystal layer. Instead, it is simply described with phase-only diffraction gratings with phase profiles having smooth edges.

The intensities of the diffraction orders for a binary phase grating and for a sinusoidal phase grating can be described with analytical relationships that depend on the phase difference $\varphi_{\max }$. For situations in between, we introduced a numerical parameter $a$ that quantifies this smoothness, which can be determined from the relative intensity of the diffraction orders. We showed that the maximum intensity that the DC order reaches in the secondary maximum expected for a phase modulation of $2 \pi$ is a very sensitive measurement and provides a very good estimation for the $a$ parameter. The results show a very good agreement with the experimental measurements, thus providing an excellent mean to quantify the fringing effect. We showed how these effects become dramatically relevant when we approach the Nyquist limit of gratings with two pixels per period. In this case the effect is not only a roundness of the phase profile, but the maximum phase shift is also reduced. We then consider a second parameter, the effective reduced maximum phase shift $\varphi^{\prime}$ $\max$, required to achieve the excellent agreement with the diffraction efficiency measurements shown in Fig. 8. The results are shown to provide results comparable with the standard convolutional approach; in fact, it can be used to calculate the broadening kernel.

These results illustrate that one must be cautious when using the diffraction grating techniques [29, 32] to calibrate the phase modulation, since the fringing effects might be affecting the diffraction efficiency and therefore leading to non-exact values.

Despite the great advances in spatial resolution achieved with the current dominant LCOS technology with respect to transmissive displays, this fringing effect is of concern because it might prevent its use when the SLM full resolution is required. This is the case for instance of our recent work [12] where we showed that fringing degrades a technique for encoding amplitude information onto phase-only holograms using Nyquist gratings. Because the gratings are not well reproduced 
due to the fringing effect, a strong DC order is originated by phase mismatch values, that overlaps on the desired response of the hologram.

We have shown these effects with a commercial LCOS-SLM from Hamamatsu, with a pixel pitch of $20 \mu \mathrm{m}$. But we expect that similar problems will affect newer higher resolution LCOS-SLMs, even more severely, as the pixel sizes decrease further. As a comparison, the transmissive CRL device used in Ref. [16] has a pixel pitch of $18 \mu \mathrm{m}$, very similar to that of the device used here, but it is not affected by these fringing effects. Although the throughput of these transmissive displays is reduced because the transparent region of the pixel is reduced relative to the pixel spacing, the absolute efficiency of the reflective LCOS devices is also reduced if one wants to have the incident beam normal to the reflective surface, because a beam splitter is required.

In summary, having a simple and rapid technique to evaluate the fringing effect and to compare different LCSLM devices and technologies is of interest to examine their performance. The technique here proposed can be very useful for this purpose and can provide quantitative values (for instance like the maximum intensity of the DC order at secondary maximum or the parameter $a$ in Eq. (2)) that could be added to the specifications of commercial devices to evaluate this effect.

\section{Acknowledgements}

Not applicable.

\section{Authors' contributions}

All coauthors contributed to the paper. IM and JAD contributed with the realization of two equivalent experimental systems in Elche and in San Diego, taking the measurements data, and analyzing them. MMSL contributed in the design of the experiments, the analysis of the results, and writing the manuscript. DMC developed the software to control the SLM and display diffractive elements. All authors read and approved the final manuscript.

\section{Funding}

IM and MMSL acknowledge financial support from Conselleria d'Educació, Investigació, Cultura i Esport; Generalitat Valenciana, Spain (PROMETEO-2017154) and from Ministerio de Ciencia, Innovación y Universidades, Spain (RTI2018-097107-B-C33).

Availability of data and materials

Not applicable.

\section{Declarations}

\section{Competing interests}

This work has no financial or non-financial competing interests.

\section{Author details}

${ }^{1}$ Instituto de Bioingeniería, Universidad Miguel Hernández de Elche, 03202 Elche, Spain. ${ }^{2}$ Departamento de Ciencia de Materiales, Óptica y Tecnología Electrónica, Universidad Miguel Hernández de Elche, 03202 Elche, Spain. ${ }^{3}$ Departamento de Física Aplicada, Universidad Miguel Hernández de Elche, 03202 Elche, Spain. ${ }^{4}$ Department of Physics, San Diego State University, San Diego, CA 92182-1233, USA.
Received: 20 August 2021 Accepted: 28 November 2021

Published online: 19 December 2021

\section{References}

1. Jullien, A.: Spatial Light Modulators. Photoniques. 101, 59-64 (2020). https:// doi.org/10.1051/photon/202010159

2. Lazarev, G., Hermerschmidt, A., Krüger, S., Osten, S.: LCOS Spatial Light Modulators: Trends and Applications, Chapter 1 in Optical Imaging and Metrology: Advanced Technologies. Wiley-VCH Verlag GmbH \& Co, KGaA (2012)

3. Schmieder, F., Klapper, S.D., Koukourakis, N., Busskamp, V., Czarske, J.W.: Optogenetic stimulation of human neural networks using fast ferroelectric spatial light modulator-based holographic illumination. Appl. Sciences. 8(7), 1180 (2018). https://doi.org/10.3390/app8071180

4. Schmieder, F., Büttner, L., Czarske, J.W.: Adaptive laser-induced ultrasound generation using a micro-mirror array spatial light modulator. Opt. Express. 24(20), 22536-22543 (2016). https://doi.org/10.1364/OE.24.022536

5. Haist, T., Osten, W.: Holography using pixelated spatial light modulators-part 1: theory and basic considerations. J. Micro/Nanolithogr MEMS MOEMS. 14(4), 041310 (2015). https://doi.org/10.1117/1.JMM.14.4.041310

6. Rosales-Guzmán, C., Forbes, A.: How to shape light with spatial light modulators. SPIE Press Bellingham. (2017). https://doi.org/10.1117/3.2281295

7. Calderón-Hermosillo, Y., García-Márquez, J., Espinosa-Luna, R., Alcalá Ochoa, N., López, V., Aguilar, A., Noé-Arias, E., Alayli, Y.: Flicker in a twisted nematic spatial light modulator. Opt. Lasers Eng. 51(6), 741-748 (2013). https://doi. org/10.1016/j.optlaseng.2013.01.013

8. Martínez, J.L.: Moreno, I, Sánchez-López, MM, Vargas, A, García-Martínez. P: Analysis of multiple internal reflections in a parallel aligned liquid crystal on silicon SLM. Opt. Express. 22(21), 25866-25879 (2014). https://doi.org/10.13 64/OE.22.025866

9. Xu, J., Qin, S.Y., Liu, C., Fu, S., Liu, D.: Precise calibration of spatial phase response nonuniformity arising in liquid crystal on silicon. Opt. Lett. 43(12), 2993-2996 (2018). https://doi.org/10.1364/OL.43.002993

10. Lingel, C., Haist, T., Osten, W.: Optimizing the diffraction efficiency of SLMbased holography with respect to the fringing field effect. Appl. Opt. 52(28), 6877-6883 (2013). https://doi.org/10.1364/AO.52.006877

11. Efron, U., Owechko, Y., Wu, S.T., Lacoe, R.C., Welkowsky, M.S., Bates, T.D., Hess, L.D.: The liquid crystal spatial light modulators: recent studies. Proc. SPIE. 567, 99-105 (1986). https://doi.org/10.1117/12.949839

12. Davis, J.A., Wolfe, E.D., Moreno, I., Cottrell, D.M.: Encoding complex amplitude information onto phase-only diffractive optical elements using binary phase Nyquist gratings. OSA Continuum. 4(3), 896-910 (2021). https://doi.org/10.1364/OSAC.418578

13. Bleha, W., Lipton, L., Wiener-Avnear, E., Grinberg, J., Reif, P., Casasent, D., Brown, H., Markevitch, B.: Application of the liquid crystal light valve to realtime optical data processing. Opt. Eng. 17(4), 174371 (1978). https://doi. org/10.1117/12.7972245

14. Liu, H.K., Davis, J.A., Lilly, R.A.: Optical data processing properties of a liquid crystal television spatial light modulator. Opt. Lett. 10(12), 635-637 (1985)

15. Kawamoto, H.: The history of liquid-crystal displays. Proc. IEEE. 90(4), 460500 (2002). https://doi.org/10.1109/JPROC.2002.1002521

16. Davis, J.A., Slovick, B.A., Tuvey, C.S., Cottrell, D.M.: High diffraction efficiency from one- and two-dimensional Nyquist frequency binary phase gratings. Appl. Opt. 47(15), 2829-2834 (2008)

17. Zhang, Z., You, Z., Chu, D.: Fundamentals of phase-only liquid crystal on silicon (LCOS) devices. Light Sci. Appl. 3(10), e213 (2014). https://doi.org/10.1 038/Isa.2014.94

18. Carbonell-Leal, M., Mendoza-Yero, O.: Effects of mitigation of pixel crosstalk in the encoding of complex fields using the double-phase method. Opt. Eng. 59(4), 041203 (2020). https://doi.org/10.1117/1.OE.59.4.041203

19. Davis, J.A., Cottrell, D.M., Campos, J., Yzuel, M.J., Moreno, I.: Encoding amplitude information onto phase-only filters. Appl. Opt. 38(23), 5004-5013 (1999). https://doi.org/10.1364/ao.38.005004

20. Clark, T.W., Offer, R.F., Franke-Arnold, S., Arnold, A.S., Radwell, N.: Comparison of beam generation techniques using a phase only spatial light modulator. Opt. Express. 24(6), 6249-6264 (2016). https://doi.org/1 0.1364/OE.24.006249

21. Efron, U., Apter, B., Bahat-Treidel, E.: Fringing-field effect in liquid-crystal beam-steering devices: an approximate analytical model. J. Opt. Soc. Am. A. 21(10), 1996-2008 (2004). https://doi.org/10.1364/JOSAA.21.001996 
22. Apter, B., Efron, U., Bahat-Treidel, E.: On the fringing-field effect in liquidcrystal beam-steering devices. Appl. Opt. 43(1), 11-19 (2004). https://doi. org/10.1364/AO.43.000011

23. Fan-Chiang, K.-H., Wu, S.-T., Chen, S.-H.: Fringing-field effects on highresolution liquid crystal microdisplays. J. Displ. Technol. 1(2), 304-313 (2005)

24. Moser, S., Ritch-Marte, M., Thalhammer, G.: Model-based compensation of pixel crosstalk in liquid crystal spatial light modulators. Opt. Express. 27(18), 25046-25063 (2019). https://doi.org/10.1364/OE.27.025046

25. Lu, T., Pivnenko, M., Robertson, B., Chu, D.: Pixel-level fringing-effect model to describe the phase profile and diffraction efficiency of a liquid crystal on silicon device. Appl. Opt. 54(19), 5903-5910 (2015). https://doi.org/10.1364/A 0.54 .005903

26. Boivier, M., Scharf, T.: Analysis of nematic-liquid-crystal binary gratings with high spatial frequency. Opt. Eng. 39(8), 2129-2137 (2000). https://doi.org/1 $0.1117 / 1.1304857$

27. Persson, M., Engström, D., Goksör, M.: Reducing the effect of pixel crosstalk in phase only spatial light modulators. Opt. Express. 20(20), 22334-22343 (2012). https://doi.org/10.1364/OE.20.022334

28. Davis, J.A., Tsai, P., Cottrell, D.M., Sonehara, T., Amako, J.: Transmission variations in liquid crystal spatial light modulators caused by interference and diffraction effects. Opt. Eng. 38(6), 1051-1057 (1999)

29. Lu, G., Zhang, Z., Wu, S., Yu, F.T.S.: Simple method for measuring phase modulation in liquid crystal televisions. Opt. Eng. 33(9), 3018-3022 (1994)

30. Goodman, J.W.: Thin Sinusoidal Phase Grating, section 4.4.4 in Introduction to Fourier Optics, 3rd edn, McGraw-Hill, New York (1968)

31. Moreno, I., Cottrell, D.M., Davis, J.A., Sánchez-López, M.M., Gutierrez, B.K.: Inphase sub-Nyquist lenslet arrays encoded onto spatial light modulators. J. Opt. Soc. Am. A. 37(9), 1417-1422 (2020). https://doi.org/10.1364/JOSAA.398828

32. Mendoza-Yero, O., Mínguez-Vega, G., Martínez-León, L., Carbonell-Leal, M., Fernández-Alonso, M., Doñate-Buendía, C., Pérez-Vizcaíno, J., Lancis, J.: Diffraction-based phase calibration of spatial light modulators with binary phase Fresnel lenses. J. Displ. Technol. 12(10), 1027-1032 (2016). https://doi. org/10.1109/JDT.2016.2580902

33. Martínez, A., Sánchez-López, M.M., Moreno, I.: Phasor analysis of binary diffraction gratings with different fill factors. Eur. J. Phys. 28(5), 805-816 (2007). https://doi.org/10.1088/0143-0807/28/5/004

34. Lizana, A., Moreno, I., Márquez, A., lemmi, C., Fernández, E., Campos, J., Yzuel, M.J.: Time fluctuations of the phase modulation in a liquid crystal on silicon display: characterization and effects in diffractive optics. Opt. Express. 16(21), 16711-16722 (2008). https://doi.org/10.1364/OE.16.016711

35. Gori, F., Santarsiero, M., Vicalvi, S., Borghi, R., Cincotti, G., di Fabrizio, E., Gentili, M.: Analytical derivation of the optimum triplicator. Opt. Commun. 157(1-6), 13-16 (1998). https://doi.org/10.1016/50030-4018(98)00518-5

36. Albero, J., Davis, J.A., Cottrell, D.M., Granger, C.E., McCormick, K.R., Moreno, I.: Generalized diffractive optical elements with asymmetric harmonic response and phase control. Appl. Opt. 52(15), 3637-3644 (2013). https://doi.org/1 0.1364/AO.52.003637

\section{Publisher's Note}

Springer Nature remains neutral with regard to jurisdictional claims in published maps and institutional affiliations.

\section{Submit your manuscript to a SpringerOpen ${ }^{\circ}$ journal and benefit from:}

- Convenient online submission

- Rigorous peer review

- Open access: articles freely available online

- High visibility within the field

- Retaining the copyright to your article

Submit your next manuscript at $\boldsymbol{\nabla}$ springeropen.com 